\title{
All-fiber laser mode-locked by the acousto-optic modulation of a fiber Bragg grating in suspended core fiber
}

Ricardo E. Silva, Tobias Tiess, Martin Becker, Tina Eschrich, Manfred Rothhardt, et al.

Ricardo E. Silva, Tobias Tiess, Martin Becker, Tina Eschrich, Manfred Rothhardt, Matthias Jäger, Alexandre A. P. Pohl, Hartmut Bartelt, "All-fiber laser mode-locked by the acousto-optic modulation of a fiber Bragg grating in suspended core fiber," Proc. SPIE 9634, 24th International Conference on Optical Fibre Sensors, 963420 (28 September 2015); doi:

$10.1117 / 12.2194929$

SPIE Event: International Conference on Optical Fibre Sensors (OFS24), 2015, Curitiba, Brazil 


\title{
All-fiber laser mode-locked by the acousto-optic modulation of a fiber Bragg grating in suspended core fiber
}

\author{
Ricardo E Silva*a,b,c ${ }^{\text {a }}$ Tobias Tiess ${ }^{\mathrm{a}, \mathrm{b}}$, Martin Becker $^{\mathrm{a}}$, Tina Eschrich ${ }^{\mathrm{a}}$, Manfred Rothhardt $\mathrm{t}^{\mathrm{a}}$, \\ Matthias Jäger ${ }^{\mathrm{a}}$, Alexandre A P Pohl ${ }^{\mathrm{c}}$ and Hartmut Bartelt ${ }^{\mathrm{a}, \mathrm{b}}$ \\ ${ }^{a}$ Leibniz Institute of Photonic Technology, IPHT, Jena, Germany \\ ${ }^{b}$ Friedrich Schiller University Jena, FSU, Jena, Germany \\ ${ }^{c}$ Federal University of Technology-Parana, UTFPR, Curitiba, Brazil
}

\begin{abstract}
An ytterbium-doped fiber laser mode-locked by the interaction of a fiber Bragg grating and longitudinal acoustic waves in a suspended core fiber is experimentally investigated. An optimized design of an acousto-optic modulator is also proposed. The results indicate output pulses with a width of less than $550 \mathrm{ps}$ at a repetition rate of $10 \mathrm{MHz}$. The reduction of the power consumed by the transducer and the grating length points out to more efficient, compact and fast acousto-optic modulators for mode-locked all-fiber lasers.
\end{abstract}

Keywords: Acousto-optic devices; microstructured fibers; fiber-optic components; fiber Bragg gratings.

\section{INTRODUCTION}

Acousto-optic modulators based on optical fibers have been successfully employed in mode-locked fiber lasers, offering an alternative solution to enable monolithic fiber setups ${ }^{1-3}$. In particular, acoustically induced mode-locking is generally based on the interaction of standing longitudinal acoustic waves and Bragg gratings, which induces amplitude modulated reflection bands at twice the acoustic frequency ${ }^{1-5}$. In standard single-mode fibers (SMF), the acoustic wave is mostly distributed over the whole fiber cross section $A_{\mathrm{s}}$, reducing the interaction between the acoustic wave and the grating in the fiber core and, consequently, the side lobe reflectivity $\eta$. This effect gets still more pronounced with increasing frequency $f$. However, acousto-optic modulators (AOM) using long gratings $L$, high acoustic powers $P_{\text {ac }}$, cladding-etched and tapered fibers, have been employed to increase the acousto-optic interaction. AOMs with a $5 \mathrm{~cm}$ grating inscribed in tapered SMF fibers have been used to produce laser pulses of $82 \mathrm{~ns}$ width at a $62.5 \mathrm{kHz}$ repetition rate for Q-switching erbium-doped fiber lasers ${ }^{6}$. Gratings of $L=12 \mathrm{~cm}$ length and voltages of $V_{\text {PZT }}=16 \mathrm{~V}$ applied to the piezoelectric transducer (PZT) have also been used in AOMs to produce mode-locking with 780 ps pulses width at a $9 \mathrm{MHz}$ repetition rate ${ }^{1}$. High voltages $V_{\text {PZT }}=20 \mathrm{~V}$ and long gratings of $L=10 \mathrm{~cm}$ have also been employed to increase the acousto-optic interaction and produce a $740 \mathrm{ps}$ pulses at $11 \mathrm{MHz}$ in mode-locked ytterbium fiber lasers ${ }^{3}$. Additionally, applying high powers to the PZT (in general, voltages higher than $10 \mathrm{~V}$ ) requires expensive power amplifiers which make the PZT electronic driver more complex and are not commonly employed in photonic labs. The reduction of the fiber diameter by means of etching or tapering techniques makes the optical properties susceptible to surface contamination and degrades the fiber mechanical stability. Moreover, the inscription of long gratings is challenging and expensive, since it requires the use of long phase masks or additional equipment to shift the fiber and the phase mask with respect to the beam, in order to avoid stitching errors ${ }^{7}$. If two beam interferometry is used, larger spatial coherence is required to expose the laser to a longer region at the fiber ${ }^{8}$.

The use of suspended core fibers (SCFs) ${ }^{9,10}$ is a good option to increase the acousto-optic modulation of Bragg gratings. SCFs feature a core suspended by silica bridges and large air holes, which reduce the silica in the cladding and, thus, increase the interaction area between the acoustic wave and the grating in the core ${ }^{11,12}$. In this study, we investigated the interaction of acoustic waves and a Bragg grating in a three holes SCF to induce mode-locking for an ytterbium-doped fiber laser. The proposed modulator design permits reduction of the grating length and the electric voltage applied to the PZT, which is desirable to reduce the size and the power consumed by acousto-optic modulators for pulsed fiber lasers.

*ricardoezq@yahoo.com.br

24th International Conference on Optical Fibre Sensors, edited by Hypolito José Kalinowski, José Luís Fabris, Wojtek J. Bock, Proc. of SPIE Vol. 9634, 963420 · @ 2015 SPIE CCC code: $0277-786 X / 15 / \$ 18 \cdot$ doi: $10.1117 / 12.2194929$ 


\section{ACOUSTO-OPTIC MODE-LOCKED FIBER LASER SETUP}

Figure 1(a) shows the $\phi=127 \mu \mathrm{m}$ diameter cross section of the SCF fabricated at the Leibniz Institute of Photonic Technology (IPHT) and used in the experiment. The fiber is $6.2 \mathrm{~cm}$ long and composed of a solid core of $d=6 \mu \mathrm{m}$ incircle diameter surrounded by three air holes of $D \sim 44 \mu \mathrm{m}$ in diameter separated by silica bridges of $t \sim 1.5 \mu \mathrm{m}$ thickness. We inscribed a $1 \mathrm{~cm}$ long grating (FBG 1) in the SCF by means of a femtosecond laser and two-beam interference, using the phase mask interferometer arrangement according to the methodology described in ${ }^{8,13}$. The grating has the Bragg wavelength at $\lambda_{\mathrm{B}}=1065.8 \mathrm{~nm}$, a $3-\mathrm{dB}$ bandwidth of $0.36 \mathrm{~nm}$ and a maximum reflectivity of $\sim 99.9 \%$. Figure 1(b) illustrates the experimental setup of the mode-locked fiber laser. A piezoelectric transducer composed of 2 layers excites the acoustic waves by means of a solid silica horn and the SCF with the inscribed FBG. The two PZT layers are electrically connected in a stack configuration to increase the mechanical displacement on the horn, and, consequently, the amplitude of the acoustic wave ${ }^{14}$. The basis of one PZT is fixed on a metallic support which is connected to an arbitrary signal generator. Conductive glue is used to make the electrical connections between the two PZTs and between one PZT and the metallic support. By using this approach, we avoided the use of conventional soldering techniques, which might cause loads on the PZT and thus reduce the strain induced by the acoustic wave in the fiber. The SCF is spliced to an SMF using an arc-discharge fusion splicer and a method to prevent the collapse of the core and the bridges. On the other side, the SCF is also spliced to the silica horn. The laser cavity is basically composed of two gratings, a delay line and the ytterbium-doped fiber to provide the gain. Figure 1(c) shows the cross section of the D-shape doped fiber fabricated at the IPHT. The fiber has a length of $4.25 \mathrm{~m}$ and a core diameter of $10 \mu \mathrm{m}$. FBG 2 is inscribed in a double-clad fiber (Nufern FUD-3950). The grating has the Bragg wavelength at $\lambda_{\mathrm{B}}=1065.8 \mathrm{~nm}$, a $3-\mathrm{dB}$ bandwidth of $0.39 \mathrm{~nm}$ and a maximum reflectivity of $\sim 50 \%$. Using a translational stage and a Peltier element to control the temperature, the reflection wavelength of FBG 2 is fine-tuned to the feedback of the induced side lobe of FBG 1 . The delay line is implemented with a standard single-mode fiber (Thorlabs 1060XP). The fiber length of $2.3 \mathrm{~m}$ is selected to match the cavity round-trip time to the reciprocal of the side lobe modulation frequency, which is twice the PZT resonance. The doped fiber is pumped by a pigtailed laser diode at $976 \mathrm{~nm}$ through a pump signal combiner. In order to precisely match the cavity length of about $10 \mathrm{~m}$ with the modulation frequency, an electrical spectrum analyzer (ESA) is used to analyze the actual cavity round trip time and adjust the length of the delay line accordingly. The shortest pulse width is observed by means of a $2.5 \mathrm{GHz}$ oscilloscope (OSC Tektronix TDS7254) and a $2 \mathrm{GHz}$ photodiode (FD), with the modulator being excited by a maximum voltage of $10 \mathrm{~V}$ and fine-tuned at $f=5.1054 \mathrm{MHz}$. Figure 1 (d) shows the laser output with a maximum amplitude power at $\lambda=1066.24 \mathrm{~nm}$ (solid curve) practically matching the induced side lobe of FBG 1 (dashed curve).
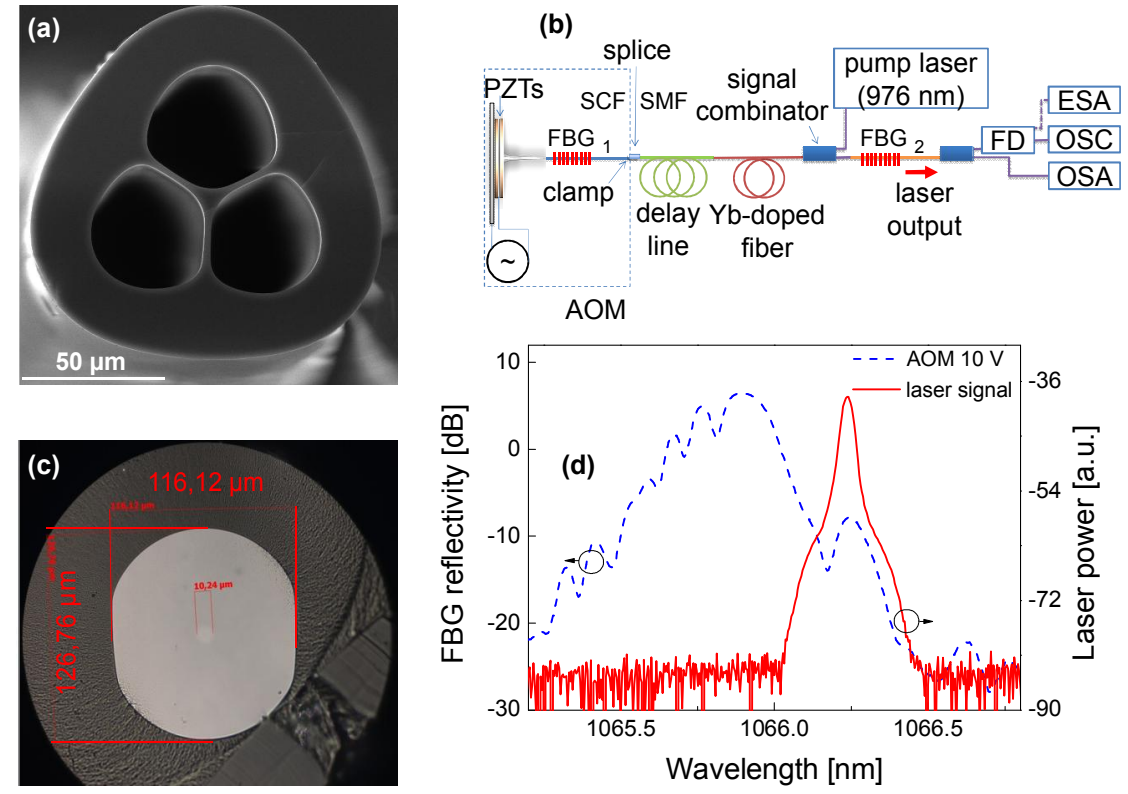

Figure 1. (a) SCF cross section used in the modulator. (b) Acousto-optic mode-locked fiber laser setup. (c) Yb-doped fiber cross section. (d) Spectra of the modulated FBG 1 (dash curve) and the laser output power (solid curve). 


\section{ACOUSTICALY MODE-LOCKED LASER PULSES}

Figure 2(a) plots the emitted pulse train of the mode-locked fiber laser generated for the $10 \mathrm{~V}$ electrical signal applied to the PZTs. The repetition rate $f_{\text {laser }}=10.2 \mathrm{MHz}$ equals twice the acoustic frequency. Figure 2(b) shows the measured trace of a single pulse with its Gaussian fit. The measured pulse width (full width at half maximum - FWHM) is less than 550 ps. However, this is close to the resolution limit of the photodiode $(2 \mathrm{GHz})$ and the oscilloscope $(2.5 \mathrm{GHz})$. Accordingly, the actual pulse width in this measurement is expected to be significantly shorter. According to the modelocking technique, the pulse width is the shorter the broader the bandwidth of the active medium is ${ }^{15}$. Although ytterbium provides a very broad gain bandwidth (FWHM $>40 \mathrm{~nm}$ ), which is desirable to produce ultra-short modelocked pulses, only a small wavelength range (axial modes) of its bandwidth is available to mode locking with acoustic waves. This is because the two feedback gratings work as narrowband spectral filters imposing strong filtering in the $\mathrm{Yb}$ bandwidth. However, since the side lobe bandwidth is described by $\lambda_{m \mathrm{FWHM}}=1.39 \lambda^{2} / \pi L n_{\text {eff }}{ }^{4}$ ( $L$ is the grating length and $n_{\text {eff }}$ the modal effective index), the use of short gratings in the modulator is expedient to increase the side lobe bandwidth and, consequently, the number of axial modes provided by the active medium to be locked by the modulator. For the $L=1 \mathrm{~cm}$ grating used in this work, the measured side lobe bandwidth is $\lambda_{m \text { ғшнм }}=200 \mathrm{pm}$, which is equivalent to $55 \mathrm{GHz}$. Since the cavity modes are spaced $10.2 \mathrm{MHz}$ apart, the modulator is able to lock a large number of axial modes ( $55 \mathrm{GHz} / 10.2 \mathrm{MHz} \sim 5400$ modes). Moreover, the lower modal effective index $n_{\text {eff }}$ observed in SCFs compared to standard fibers also contributes to increasing the side lobe bandwidth $\lambda_{m \mathrm{FWHM}}$ and reducing the pulse width. For the SCF investigated, the effective index is estimated at $n_{\text {eff }}=1.43$. However, the best results of pulse width observed in the $500-$ 600 ps range are still far away from the theoretical lower limit around $0.315 / 55 \mathrm{GHz}=6 \mathrm{ps}$, if we assume a transform limited laser pulse with an almost Gaussian shape in the time domain ${ }^{3}$. This is because the pulse width also strongly depends on other modulator and fiber cavity parameters which are not investigated in this work (e.g. modulation depth, dispersion of the fibers that compose the cavity and the active medium). The modulation depth is a parameter related to the maximum and minimum reflectivity $R_{\max }$ and $R_{\min }$ of the side lobe calculated as $\left(R_{\max }-R_{\min }\right) / R_{\max }{ }^{2}$. An increase in modulation depth reduces the pulse width ${ }^{16}$, which is obtained by the application of high powers to the PZT, an improvement of the modulator design or the use of SCFs with larger air holes. However, the maximum modulation depth achieved for the proposed modulator is about $11 \mathrm{~dB}$ as shown in Fig. 3. Although we did not measure the dispersion of the laser cavity, the use of fibers with high anomalous dispersion is also desirable to narrow the pulse width, and in this sense, the use of the SCFs is expedient also for this purpose.
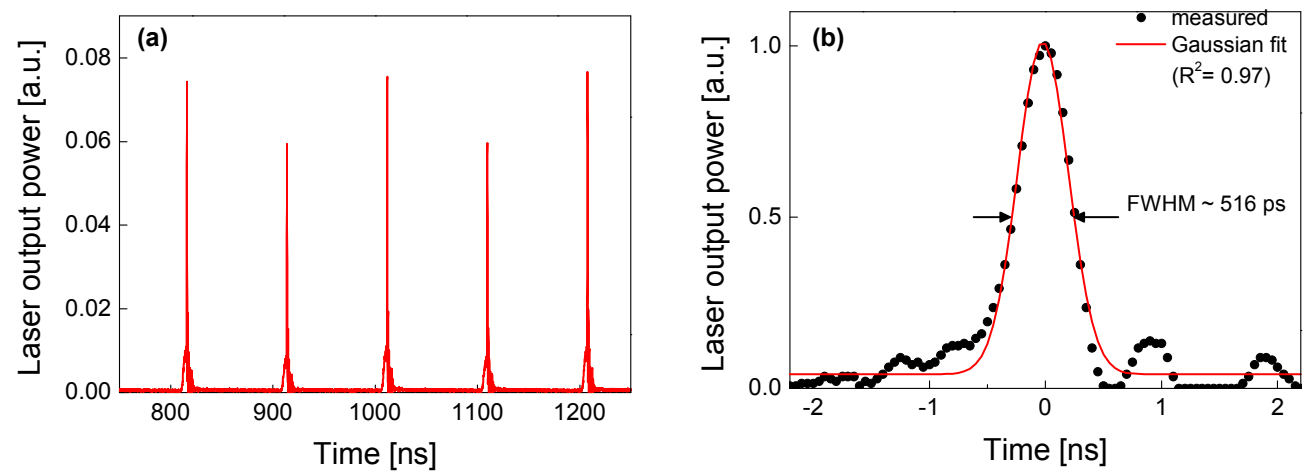

Figure 2. (a) Mode-locked fiber laser pulse train with repetition rate of $10.2 \mathrm{MHz}$ generated when a $10 \mathrm{~V}$ electrical signal is applied to the PZTs at $f=5.1054 \mathrm{MHz}$. (b) Measurement of a single laser pulse with a width of less than $550 \mathrm{ps}$ (FWHM).

\section{CONCLUSION}

In conclusion, the acousto-optic modulation of a $1 \mathrm{~cm}$ FBG using longitudinal acoustic waves in a suspended core fiber is investigated, and its application to modulate a mode-locked ytterbium doped fiber laser is demonstrated. The modulated side lobe reflectivity is $11 \mathrm{~dB}$ higher than the grating without modulation and has a 3-dB bandwidth of $200 \mathrm{pm}$. The SCF exhibits a higher modulation efficiencies compared to a solid SMF, which is useful to reduce the modulator size and the energy required to excite the acoustic waves. Moreover, the SCF provides better mechanical 
stability compared to fiber taper and etching techniques. For the best results, with a signal of $10 \mathrm{~V}$ applied to the modulator, the fiber laser emitted pulses with a width of $\sim 550 \mathrm{ps}$ at a repetition rate of $10.2 \mathrm{MHz}$. This shows that active mode-locking with acoustically modulated fiber Bragg gratings in a suspended core fiber is an efficient and well applicable method for the generation of laser pulses. In addition, the reduction of grating length and of the power consumed by the modulator indicate possibilities of more efficient acousto-optic modulators for all-fiber lasers.

\section{ACKNOWLEDGMENTS}

Funding by the Thuringian Ministry of Education, Science and Culture (EFRE program) is gratefully acknowledged. This study was also supported in part by the Cordenação de Aperfeiçoamento de Pessoal de Nível Superior (CAPES), Ministério da Defesa - Projeto Pró-Defesa, and CNPq/FAPESP - INCT (FOTONICOM).

\section{REFERENCES}

[1] Cuadrado-Laborde, C., Diez, A., Delgado-Pinar, M., Cruz, J. L.., Andrés, M. V., "Mode locking of an all-fiber laser by acousto-optic superlattice modulation,” Opt. Lett. 34(7), 1111 (2009).

[2] Cuadrado-Laborde, C., Díez, A., Cruz, J. L.., Andrés, M. V., "Experimental study of an all-fiber laser actively mode-locked by standing-wave acousto-optic modulation," Appl. Phys. B 99(1-2), 95-99 (2009).

[3] Villegas, I. L., Cuadrado-Laborde, C., Abreu-Afonso, J., Díez, A., Cruz, J. L., Martínez-Gámez, M. A., Andrés, M. V., "Mode-locked Yb-doped all-fiber laser based on in-fiber acoustooptic modulation," Laser Phys. Lett. 8(3), 227-231 (2011).

[4] Liu, W. F., Russell, P. S. J.., Dong, L., "100\% efficient narrow-band acoustooptic tunable reflector using fiber Bragg grating,” J. Light. Technol. 16(11), 2006-2009 (1998).

[5] Silva, R. E., Franco, M. A. R., Neves, P. T., Bartelt, H.., Pohl, A. A. P., "Detailed analysis of the longitudinal acousto-optical resonances in a fiber Bragg modulator.," Opt. Express 21(6), 6997-7007(2013).

[6] Delgado-Pinar, M., Zalvidea, D., Diez, a., Perez-Millan, P.., Andres, M., "Q-switching of an all-fiber laser by acousto-optic modulation of a fiber Bragg grating.," Opt. Express 14(3), 1106-1112 (2006).

[7] Hill, K. O., Meltz, G., "Fiber Bragg grating technology fundamentals and overview,” J. Light. Technol. 12631276 (1997).

[8] Becker, M., Bergmann, J., Brückner, S., Franke, M., Lindner, E., Rothhardt, M. W.., Bartelt, H., "Fiber Bragg grating inscription combining DUV sub-picosecond laser pulses and two-beam interferometry," Opt. Express 16(23), 19169 (2008).

[9] Phan Huy, M. C., Laffont, G., Dewynter, V., Ferdinand, P., Roy, P., Auguste, J.-L., Pagnoux, D., Blanc, W.., Dussardier, B., "Three-hole microstructured optical fiber for efficient fiber Bragg grating refractometer," Opt. Lett. 32(16), 2390 (2007).

[10] Afshar V., S., Warren-Smith, S. C.., Monro, T. M., "Enhancement of fluorescence-based sensing using microstructured optical fibres," Opt. Express 15(26), 17891 (2007).

[11] Silva, R., Hartung, A., Rothhardt, M., Pohl, A. A.., Bartelt, H., "Simulation of acousto-optical modulation of fiber Bragg gratings in suspended core fibers," Work. Spec. Opt. Fibers their Appl., F2.22, OSA, Washington, D.C. (2013).

[12] Silva, R. E., Becker, M., Hartung, A., Rothhardt, M., Pohl, A. A. P.., Bartelt, H., "Reflectivity and bandwidth modulation of fiber Bragg gratings in a suspended core fiber by tunable acoustic waves," IEEE Photonics J. 6(6), 1-8 (2014).

[13] Becker, M., Fernandes, L., Rothhardt, M., Bruckner, S., Schuster, K., Kobelke, J., Frazao, O., Bartelt, H.., Marques, P., "Inscription of fiber Bragg grating arrays in pure silica suspended core fibers," IEEE Photonics Technol. Lett. 21(19), 1453-1455 (2009).

[14] Wang, X., Ehlers, C.., Neitzel, M., "Electro-mechanical dynamic analysis of the piezoelectric stack," Smart Mater. Struct. 5(4), 492-500 (1996).

[15] Kuizenga, D.., Siegman, A., "FM and AM mode locking of the homogeneous laser - Part I: Theory," IEEE J. Quantum Electron. 6(11), 694-708 (1970).

[16] Li, Y., Lou, C., Han, M.., Gao, Y., "Detuning characteristics of the AM mode-locked fiber laser," Opt. Quantum Electron. 33(6), 589-597 (2001). 